\title{
Evaluation of Cortical Thickness after Traumatic Brain Injury in Military Veterans
}

\author{
Alex P. Michael, ${ }^{1, *}$ Jeffrey Stout, ${ }^{2, *}$ P. Tyler Roskos, ${ }^{3}$ Jacob Bolzenius, ${ }^{4}$ Jeffrey Gfeller, ${ }^{5}$ \\ David Mogul, ${ }^{2}$ and Richard Bucholz ${ }^{6}$
}

\begin{abstract}
Military service members frequently sustain traumatic brain injuries (TBI) while on active duty, a majority of which are related to explosive blasts and are mild in severity. Studies evaluating the cortical gray matter in persons with injuries of this nature remain scarce. The purpose of this study was to assess cortical thickness in a sample of military veterans with chronic blast-related TBI. Thirty-eight veterans with mild TBI and 17 veterans with moderate TBI were compared with 58 demographically matched healthy civilians. All veterans with TBI sustained injuries related to a blast and were between 5 and 120 months post-injury $(M=62.08)$. Measures of post-traumatic stress disorder (PTSD) and depression were administered, along with a battery of neuropsychological tests to assess cognition. The Freesurfer software package was used to calculate cortical thickness of the participants. Results demonstrated significant clusters of cortical thinning in the right hemispheric insula and inferior portions of the temporal and frontal lobe in both mild and moderate TBI participants. The TBI sample from this study demonstrated a high incidence of comorbid PTSD and depression symptoms, which is consistent with the previous literature. Cortical thickness values correlated with measures of PTSD, depression, and postconcussive symptoms. This study provides evidence of cortical thinning in the context of chronic blast-related mild and moderate TBI in military veterans who have comorbid psychiatric symptoms. Our findings provide important insight into the natural progression of long-term cortical change in this population and may have implications for future clinical evaluation and treatment.
\end{abstract}

Key words: cortical thickness; magnetic resonance imaging; military veterans; traumatic brain injury

\section{Introduction}

$\mathbf{M}$ ILITARY SERVICE MEMBERS engaged in modern conflicts frequently sustain traumatic brain injury (TBI) while on active duty. Recent studies have estimated that more than $20 \%$ of military veterans from Operations Enduring Freedom and Iraqi Freedom (OEF/OIF) return from combat exhibiting symptoms consistent with TBI, the majority of which are mild in severity. ${ }^{1,2}$ The diagnosis and management of mild TBI in the military population has been a particularly unique challenge to clinicians, because a significant portion of these injuries occur in the context of explosive blasts, as opposed to the traditional contact-induced TBI seen in the civilian population. ${ }^{3}$ Regardless of etiology, diagnosis of mild TBI is currently restricted to self-reported and observed symptoms.

Blast-related TBI may occur from a combination of several injury mechanisms. The detonation of an explosive device causes the rapid expansion of a solid or liquid into the gas phase forming a pressure wave. This wave then interacts with air- or fluid-filled body organs, such as the brain, inducing a sudden increase in intracranial pressure and resulting in penetration of brain tissue, disruption of axonal pathways, and damage to capillaries. ${ }^{4-6}$ These blast-induced effects are referred to as primary injury. ${ }^{5,7,8}$ The blast wave may also propel objects toward a person causing secondary injury or it may force the person into other solid objects (i.e., tertiary injury). While secondary and tertiary injury are also consistent with contact-induced TBI, primary injury is generally unique to blast events and may have independent consequences on brain tissue structure and function.

Mild TBI is thought to result from damage to both cortical tissue and white matter tracts through a combination of direct tissue compression, hemorrhage, and axonal injury. ${ }^{8-12}$ Conventional neuroimaging using CT or MRI is typically normal after blast-related mild TBI, likely because of the microscopic nature of the injury. ${ }^{13,14}$ Despite the lack of positive findings, more than $50 \%$ of veterans meet

\footnotetext{
${ }^{1}$ Division of Neurosurgery, Southern Illinois University School of Medicine, Springfield, Illinois.

${ }^{2}$ Department of Biomedical Engineering, Illinois Institute of Technology, Chicago, Illinois.

${ }^{3}$ Department of Physical Medicine and Rehabilitation, Oakwood, Wayne State University, School of Medicine, Dearborn, Michigan.

${ }^{4}$ University of Missouri-St. Louis, St. Louis, Missouri.

Departments of ${ }^{5}$ Psychology and ${ }^{6}$ Neurosurgery, Saint Louis University School of Medicine, St. Louis, Missouri.

*The first two authors contributed equally to this article.
} 
the criteria for post-concussive disorder at long-term follow-up postinjury, suggesting a persistent neural injury. ${ }^{15}$ Although the disruption of white matter integrity has been investigated after mild TBI both in civilians and blast-injured veterans, ${ }^{3,16,17}$ there remains a paucity of neuroimaging studies evaluating cortical gray matter, especially after blast-related injury.

Surface based morphometry (SBM) is a well-established method of evaluating the thickness of the cortical gray matter from magnetic resonance images. ${ }^{18-20} \mathrm{SBM}$ has detected cortical thinning in pediatric patients and patients with severe TBI, ${ }^{21-25}$ although examinations of mild TBI and blast-related TBI remain underrepresented in brain morphometric research. A previous examination of cortical thickness after blast-related TBI in the military reported thinning in the left superior temporal and superior frontal gyri. ${ }^{26}$ This study did not assess changes in cortical thickness beyond the acute phase of injury and was limited by sample size. Cortical thickness measurements in persons with post-acute TBI would allow for further elucidation of the potential neuropathology associated with TBI and to evaluate long-term cortical changes related to blast injury.

A high incidence of comorbid post-traumatic stress disorder (PTSD) has also been documented in military personnel with blastrelated injuries. A study by Hoge and colleagues ${ }^{2}$ found that $9.1 \%$ of veterans returning from OEF/OIF met criteria for PTSD. This incidence rose to $43.9 \%$ in those veterans who experienced mild TBI with loss of consciousness (LOC), suggesting that physical injury increases vulnerability to psychological stress. Further, PTSD severity in the military population has been found to inversely correlate with cortical thickness independent of TBI. ${ }^{27}$ This study also found evidence for an additive effect of comorbid mild TBI and PTSD associated with additional areas of cortical thinning.

Blast-related TBI has been linked to a variety of cognitive and behavioral difficulties, although the severity of these impairments in relation to cortical changes remains unclear. Cognitive functions such as learning, memory, attention, and executive function have all been shown to relate with blast-related TBI. ${ }^{27-30}$ Performance on neuropsychological measures in these domains has also been linked to differences in neural activity in veterans with mild TBI as measured by magnetoencephalography (MEG). ${ }^{16,31,32}$ These changes are thought to result from disruptions in the integrity of neural networks in both cortical and subcortical areas. ${ }^{32}$

Thus far, cortical thinning has been shown to correlate with performance on measures of verbal memory and visuomotor speed, which are common cognitive domains affected in persons with TBI. ${ }^{33}$ No studies, however, have investigated these factors in relation to each other in a sample of veterans with blast-related injuries. ${ }^{33}$

The present study sought to enhance our understanding of the effects that TBI has on cortical gray matter by investigating changes in cortical thickness during the chronic phase of blastrelated TBI. We further assessed the relationship between severity of injury and cortical thickness by comparing mild and moderate blast-related TBI groups. We expected TBI participants to exhibit decreased cortical thickness (i.e., cortical thinning) compared with demographically matched healthy controls. Further, it was expected that a "dose-dependent" relationship would exist between injury severity and the degree of cortical thinning (moderate TBI cortical thinning >mild TBI cortical thinning).

We also investigated the relationship between cortical thinning and scores on neuropsychological tests, as well as measures of PTSD and depression symptoms. Based on previous literature, it was expected that cortical thinning would be related to poorer performance on neuropsychological tests, as well as severity of psychiatric symptom reporting.

\section{Methods}

\section{Participants}

Seventy-one military veterans with TBI were recruited from primarily urban areas across the United States as part of a larger ongoing study. Participants were excluded from the study if they were: (1) nonfluent English speakers, (2) an undocumented alien, incarcerated, or active duty military, (3) under age 18, (4) intellectually disabled, or (5) if they had a pre-existing condition that would preclude standard administration of study procedures, such as deafness or blindness. All veterans had sustained a TBI at a minimum of 5 months before enrollment in the study.

All participants signed an informed consent document and were enrolled in the study for 2 days. On day 1, a research assistant administered a semistructured diagnostic interview to collect demographic, medical, psychiatric, and injury history information. In addition, participants were evaluated for current level of disability using the Disability Rating Scale (DRS) and the Community Integration Questionnaire (CIQ), which were also administered by a research assistant. Neuroimaging studies, including structural MRI, were conducted on the second day of study participation.

The Defense and Veterans Affairs consensus definition of TBI was used to classify injury severity based on participant injury characteristics (duration of LOC, duration post-traumatic amnesia, duration of confusion, initial imaging findings, and initial Glasgow Coma Scale scores, when available). ${ }^{34}$ The injury severity data collected during interview with the participants was verified via review of medical records when possible. Based on these criteria, all military veterans had sustained a nonpenetrating mild or moderate blast-related TBI. Research has suggested a high comorbidity of psychiatric and neurological disorders in patients with TBI; therefore, these disorders were screened for but not used as ex-

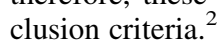

Sixty-two healthy persons were also recruited from the St. Louis metropolitan area to create a demographically matched healthy control group for comparison. Additional exclusion criteria for the healthy control group included history of psychiatric or neurological illness, substance use disorder, and other cognitive or developmental disorders.

\section{Neuropsychological evaluation}

The following neuropsychological tests were administered as part of a larger battery and included in analyses: the Digit Span subtest from Wechsler Adult Intelligence Scale Third Edition (WAIS-III DS), ${ }^{35}$ Trail Making Test Part A (TMT A), Trail Making Test Part B (TMT B), ${ }^{36}$ the California Verbal Learning Test Second Edition (CVLT-II) Total Recall and Long Delay Free Recall trial scores ${ }^{37}$ and Condition 1 from the Verbal Fluency subtest from the Delis Kaplan Executive Functioning System (DKEFS VF1). ${ }^{38}$ The Neurobehavioral Symptoms Inventory (NSI) ${ }^{39}$ was administered to assess for post-concussive symptoms.

In addition, the Patient Health Questionniare-9 (PHQ-9) was used to assess for comorbid depression, and the PTSD Checklist Civilian version (PCL-C) was given to assess symptoms of PTSD. ${ }^{40,41}$ A trained research assistant administered all tests, and raters were blind to the participants' grouping at the time of testing. Analysis of variance (ANOVA) statistical analyses were also performed to identify differences between healthy controls and the TBI groups on neuropsychological measures.

\section{MRI data acquisition and cortical thickness assessment}

T1-weighted MRI data with 1-mm isotropic resolution was acquired on all subjects using a Phillips Achieva 3-Telsa MRI scanner with an 8-channel birdcage headcoil. T1 images were acquired using a turbo field echo scan with an 8 degree flip angle and sense 
parallel acquisition of factor 2. Semiautomated cortical segmentation was performed using Freesurfer v5.0.0 software (Athinoula A. Martinos Center for Biomedical Imaging, Charlestown, MA; http://surfer.nmr.mgh.harvard.edu) as described by Dale and associates. $^{18}$

Tissue classification into gray matter (GM), white matter (WM), and cerebrospinal fluid (CSF) was calculated using signal intensity differences and known previous spatial statistics. ${ }^{42,43}$ Pial and WM surfaces were constructed using a meshing algorithm of classified voxels. ${ }^{20,44}$ At each node, the cortical thickness was estimated from the calculated minimal distance between the pial and WM surface meshes. ${ }^{19}$ Automated registration of major cortical landmarks and spatial warping was performed to optimize curvature similarities of the cortical alignment with the surface atlas. ${ }^{45}$ Once aligned and warped to the atlas space, the surfaces were labeled according to known anatomical regions. ${ }^{46}$

Two independent researchers visually checked the pial and WM surfaces created for each subject. Any errors in tissue classification that occurred during the automated processing were manually edited and rerun through the Freesurfer processing pipeline. In addition, the MRIs were scored for image quality. Low image quality scans were more likely to have poor reconstructions, and seven subjects (two healthy controls, four mild TBI, one moderate TBI) were removed for this reason. Two subjects ( 1 mild TBI and 1 moderate TBI) were removed because of uncorrectable cortical reconstructions, and two healthy controls were removed because of mild ventricular dilation on radiological review. A total of nine military veteran subjects were also excluded because their primary mechanism of injury was not blast-related (e.g., assault, motor vehicle accident). Fifty-five military veterans with TBI (54 male, 1 female) and 58 healthy controls ( 54 male, 4 female) made up the final study sample.

\section{Cortical thickness statistics}

Cortical thickness maps were convolved with a two-dimensional 15-mm Gaussian surface smoother. The data were imported into the QDec general linear modeling interface included with Freesurfer and analyzed using a different offset different slope model. To address familywise error, the Monte Carlo null- $Z$ method was used to correct for multiple comparisons. ${ }^{47} \mathrm{~A}$ one-sided null- $Z$ correction at $p<0.05$ was used to test the hypothesis of cortical thinning in the TBI groups.

\section{Correlation between neuropsychological measures and significant cortical thickness cluster}

To investigate the relationship between neuropsychological measures and cortical thickness values, the mild and moderate TBI groups were combined, and statistical analyses were performed in comparison with the healthy control group. The resulting familywise error corrected cluster was traced and generated into a subject label in QDec. The average cortical thickness was fit for each subject using the Freesurfer command, mris anatomical_fit, isolated to the $p<0.05$ cluster region of interest (ROI). The average cortical thickness over the ROI was parsed out of each file using python and compiled into a text file, which was imported into SPSS to calculate Pearson correlations with neuropsychological test scores, as well as measures of depression and PTSD.

\section{Results}

\section{Demographic and clinical characteristics}

Of the participants with TBI, 38 had sustained mild TBI and 17 sustained moderate TBI. No significant differences were observed between the groups for mean age, education, or frequency of sex
Table 1. Demographic and Clinical Characteristics for the Healthy Control, Mild Traumatic Brain Injury, and Moderate Traumatic Brain Injury Groups

\begin{tabular}{|c|c|c|c|}
\hline Variable & $\begin{array}{l}\text { Healthy } \\
\text { controls } \\
(\mathrm{n}=58)\end{array}$ & $\begin{array}{c}\text { Mild } \\
\text { TBI } \\
(\mathrm{n}=38)\end{array}$ & $\begin{array}{c}\text { Moderate } \\
\text { TBI } \\
(\mathrm{n}=17)\end{array}$ \\
\hline \multicolumn{4}{|l|}{ Sex } \\
\hline Male & 54 & 37 & 17 \\
\hline Female & 4 & 1 & 0 \\
\hline \multicolumn{4}{|l|}{ Ethnicity $^{1}$} \\
\hline Caucasian & 27 & 36 & 14 \\
\hline African American & 24 & 1 & 1 \\
\hline Hispanic & 1 & 1 & 1 \\
\hline Asian/Pacific Islander & 4 & 0 & 0 \\
\hline Other & 2 & 0 & 1 \\
\hline \multicolumn{4}{|l|}{ Duration LOC ${ }^{2}$} \\
\hline$>24 \mathrm{~h}$ & - & 0 & 2 \\
\hline $30 \mathrm{~min}$ to $24 \mathrm{~h}$ & - & 0 & 3 \\
\hline $0-30 \mathrm{~min}$ & - & 22 & 10 \\
\hline Unknown/unavailable & - & 16 & 2 \\
\hline \multicolumn{4}{|l|}{ Duration of PTA ${ }^{3}$} \\
\hline$>24 h$ & - & 14 & 14 \\
\hline $0-24 \mathrm{~h}$ & - & 21 & 1 \\
\hline Unknown/unavailable & - & 3 & 2 \\
\hline \multicolumn{4}{|l|}{ Initial CT scan ${ }^{4}$} \\
\hline Positive & - & 1 & 7 \\
\hline Negative & - & 9 & 3 \\
\hline Unknown/unavailable & - & 28 & 7 \\
\hline \multicolumn{4}{|l|}{ DRS Score } \\
\hline 0 & - & 23 & 5 \\
\hline 0.5 & - & 1 & 1 \\
\hline 1 & - & 5 & 2 \\
\hline 2 & - & 2 & 3 \\
\hline 3 & - & 2 & 3 \\
\hline 4 & - & 3 & 2 \\
\hline 5 & - & 2 & 1 \\
\hline
\end{tabular}

TBI, traumatic brain injury; LOC, loss of consciousness; PTA, posttraumatic amnesia; CT, computed tomography; DRS, disability rating scale.

${ }^{1}$ Pearson $\chi^{2}=32.41, p<0.001 ;{ }^{2}$ Pearson $\chi^{2}=15.31 ; p<0.01 ;{ }^{3}$ Pearson $\chi^{2}=12.29 ; p<0.01 ;{ }^{4}$ Pearson $\chi^{2}=14.24 ; p<0.01$.

Entries are numbers of participants, unless otherwise specified.

(Table 1). The mild TBI group was found to range from age 23 to 52 years (range 29), moderate from 25 to 62 years (range 37), and civilian control from 18 to 54 (range 36). All veterans with TBI sustained injuries related to a blast and were between 5 and 120 months post-injury $(M=62.08)$. The moderate TBI group showed greater incidence and duration of LOC $\left(\chi^{2}=15.31, p<0.01\right)$ and post-traumatic amnesia $\left(\chi^{2}=12.29, p<0.01\right)$ compared with the mild TBI group.

The findings from initial neuroimaging (CT scan) were largely unavailable or unknown. Patients with more moderate TBI had known abnormalities on the initial CT scan compared with participants with mild TBI. The mild and moderate TBI groups did not show differences in the frequency of scores on the DRS nor were there statistically significant differences between groups on the CIQ. Both groups scored lower on the CIQ compared with the control group, indicating that both TBI groups had a relatively similar level of current disability. See Tables 1 and 2 for descriptive statistics and further results from these analyses. 
Table 2. Means and Standard Deviations for Demographic and Neuropsychological Measures

\begin{tabular}{|c|c|c|c|c|c|c|c|c|c|}
\hline \multirow[b]{2}{*}{ Variable } & \multicolumn{3}{|c|}{ Healthy Controls } & \multicolumn{3}{|c|}{ Mild TBI } & \multicolumn{3}{|c|}{ Moderate TBI } \\
\hline & $M$ & $S D$ & $N$ & $M$ & $S D$ & $N$ & $M$ & $S D$ & $N$ \\
\hline Age & 30.53 & 8.70 & 58 & 32.79 & 7.26 & 38 & 34.41 & 8.95 & 17 \\
\hline Education (years) & 14.74 & 2.43 & 58 & 13.82 & 2.08 & 38 & 14.18 & 1.91 & 17 \\
\hline Months Post-Injury & - & - & - & 62.08 & 23.91 & 38 & 70.41 & 20.41 & 17 \\
\hline $\mathrm{NSI}^{1}$ & $5.14^{\mathrm{a}}$ & 8.05 & 58 & $35.05^{\mathrm{b}}$ & 14.96 & 38 & $49.18^{\mathrm{c}}$ & 14.56 & 17 \\
\hline $\mathrm{CIQ}^{2}$ & $21.23^{\mathrm{a}}$ & 6.29 & 58 & $17.28^{\mathrm{b}}$ & 5.57 & 38 & $15.65^{\mathrm{b}}$ & 5.85 & 17 \\
\hline PCL-C ${ }^{3}$ & $23.16^{\mathrm{a}}$ & 6.34 & 57 & $48.51^{\mathrm{b}}$ & 15.26 & 37 & $58.00^{\mathrm{c}}$ & 16.31 & 17 \\
\hline PHQ-9 ${ }^{4}$ & $1.80^{\mathrm{a}}$ & 2.03 & 57 & $9.81^{\mathrm{b}}$ & 5.48 & 36 & $12.94^{\mathrm{c}}$ & 6.23 & 17 \\
\hline WAIS-III Digit Span & $18.47^{\mathrm{a}}$ & 3.78 & 58 & $16.16^{\mathrm{b}}$ & 3.48 & 38 & $15.65^{\mathrm{b}}$ & 3.92 & 17 \\
\hline TMT A & 27.67 & 10.42 & 58 & 27.24 & 8.79 & 37 & 27.18 & 11.59 & 17 \\
\hline TMT B & 67.16 & 37.10 & 58 & 64.57 & 22.78 & 37 & 75.12 & 25.39 & 17 \\
\hline CVLT-II 1-5 Total & 46.95 & 7.54 & 58 & 45.35 & 10.80 & 37 & 45.88 & 11.43 & 17 \\
\hline CVLT-II LDFR & 10.29 & 2.66 & 58 & 9.51 & 3.95 & 37 & 9.06 & 4.15 & 17 \\
\hline DKEFS VF ${ }^{6}$ & $43.50^{\mathrm{a}}$ & 11.04 & 58 & $36.73^{\mathrm{b}}$ & 11.70 & 37 & $35.00^{\mathrm{b}}$ & 9.35 & 17 \\
\hline
\end{tabular}

TBI, Traumatic Brain Injury; M, mean; SD, standard deviation; NSI, Neurobehavioral Symptom Inventory. CIQ, Community Integration Questionnaire; PCL-C, Post-Traumatic Stress Disorder Checklist Civilian version; PHQ-9, Patient Health Questionniare-9; WAIS-III, Wechsler Adult Intelligence Scale Third Edition; TMT A, Trail Making Test Part A; TMT B, Trail Making Test Part B; CVLT-II, California Verbal Learning Test Second Edition; LDFR, Long Delay Free Recall; DKEFS, Delis Kaplan Executive Functioning System; VF1, Verbal Fluency Condition 1.

${ }^{1}$ Analysis of variance (ANOVA) $\mathrm{F}_{(2,109)}=127.22, p<0.001 ;{ }^{2}$ ANOVA $\mathrm{F}_{(2,110)}=8.23, p<0.001 ;{ }^{3}$ ANOVA $\mathrm{F}_{(2,108)}=84.58 ; p<0.001 ;{ }^{4}$ ANOVA $\mathrm{F}_{(2,107)}=66.07 ; p<0.001 ;{ }^{5}$ ANOVA $\mathrm{F}_{(2,110)}=6.37 ; p<0.01 ;{ }^{6}$ ANOVA $_{(2,109)}=6.29 ; p<0.01$.

a,b,c Means with different superscripts are statistically significantly different, $p<0.05$.

\section{Cortical thickness group analyses}

Several clusters of cortical thinning were found when comparing the healthy controls with TBI participants. The output statistical maps are displayed in Figure 1. After null-Z familywise error rate correction, only one cluster encompassing the right inferior temporal, right insula, and right inferior frontal areas remained statistically significant $\left(t[93]=4.15, p=0.00008\right.$; cluster size $\left.=7844 \mathrm{~mm}^{2}\right)$.

No areas in the left hemisphere exhibited significant cortical thinning. The resulting clusters can be seen in Figure 2A. Null-Z corrected statistical comparison between controls and moderate TBI highlighted a cluster of cortical thinning in the inferior temporal area $\left(t[72]=4.53 ; p=0.00011\right.$; cluster size $\left.=5393 \mathrm{~mm}^{2}\right)$; see Figure $2 \mathrm{~B}$. The combined grouping of mild and moderate TBI compared with healthy controls resulted in a larger cluster over the inferior frontal, temporal, and insula regions $(t[109]=3.84$; $p=0.0002$, cluster size $=120678 \mathrm{~mm}^{2}$ ); see Figure 2C. Within this cluster, mild and moderate TBI participants were compared but were not statistically different at the significance level of $p<0.01$ $(t[54]=0.37 ; p=0.72)$. The average cortical thickness in the region of significance for each subject group is graphically represented in Figure 3.

\section{Neuropsychological analyses}

Results from ANOVA with Tukey honest significant difference (HSD) post hoc analyses involving the neuropsychological measures are provided in Table 2. Generally, results showed that the control group scored higher than the mild and moderate TBI groups on WAIS-III digit span and on VF1 from the DKEFS. Scores were not significantly different between groups on other included measures of cognitive functioning. The moderate TBI group reported a greater number of post-concussive symptoms compared with the mild TBI group as measured using the NSI total score. Both TBI groups reported a greater number of post-concussive symptoms compared with the control group (moderate TBI scored higher compared with mild TBI). With regard to symptoms of depression and PTSD, ANOVA with Tukey HSD post hoc analyses showed that the moderate TBI group reported the highest number of clinically significant symptoms. The mild TBI group also showed higher symptom reporting compared with the control group.

We also calculated two-tailed Pearson correlational coefficients between neuropsychological tests scores, scores on measures of depression and PTSD, and average cortical thickness cluster scores. Results showed statistically significant correlations between

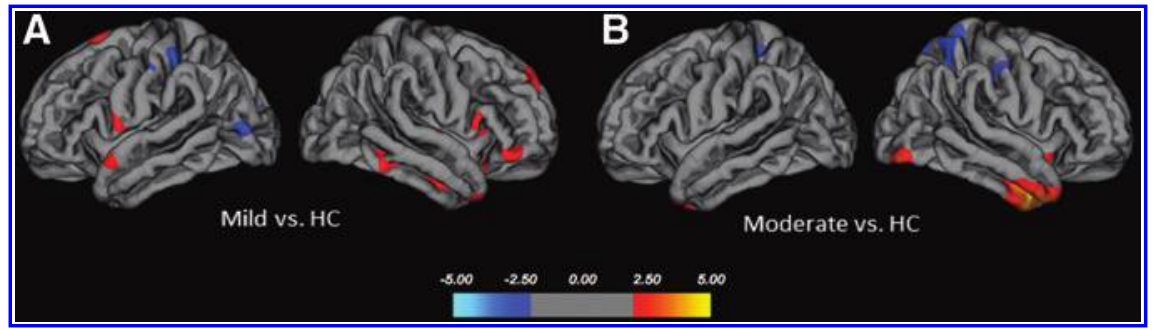

FIG. 1. T-test results between healthy controls (HC) and participants with mild traumatic brain injury (TBI) (A) and moderate TBI (B) displayed on the Freesurfer average brain. Red represent greater thickness in HCs, and blue represents greater thickness in subjects with TBI. Color image is available online at www.liebertpub.com/neu 


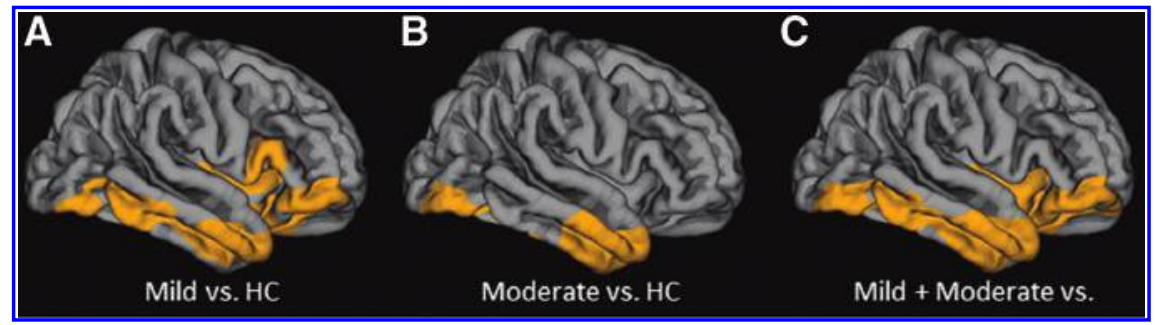

FIG. 2. Cortical thickness comparison between healthy controls (HC) and blast-induced participants with traumatic brain injury (TBI). Display of the resulting threshold corrected clusters to the familywise error rate of $p<0.05$ the using null-Z method. The group comparisons are $\mathrm{HC}$ versus mild TBI (A), HC versus moderate TBI (B), and HC versus mild + moderate TBI (C). Color image is available online at www.liebertpub.com/neu

cortical thickness cluster scores and the NSI total scores $(\mathrm{r}=-$ $0.330, p<0.001)$, DKEFS VF1 $(r=0.212, p<0.05)$, PHQ-9 total scores $(r=-0.251, p<0.01)$, and PCL-C total scores $(r=-0.249$, $p<0.01)$. Correlations between cortical thickness clusters and other neuropsychological measures were nonsignificant.

After applying Bonferroni correction to the significant correlations to control for familywise error (significant threshold of $p<0.005$ ), only the NSI total score correlated significantly with the cortical thickness cluster values. These results suggest that cortical thickness values had limited low magnitude inverse correlation with post-concussive syndrome symptoms and other aspects of emotional and behavioral functioning, as well as a possible direct relationship with a cognitive measure. The exact implications of these findings are unclear, however, because of their statistical nonsignificance when using the most conservative interpretive criterion.

\section{Discussion}

In the current study, healthy civilian controls were compared with military veterans with mild or moderate blast-related TBI using the SBM method. To our knowledge, this is the first such study to examine cortical thickness in a sample of veterans with chronic blast-related TBI. As expected, we found statistically significant cortical thinning in multiple areas, including the right in-

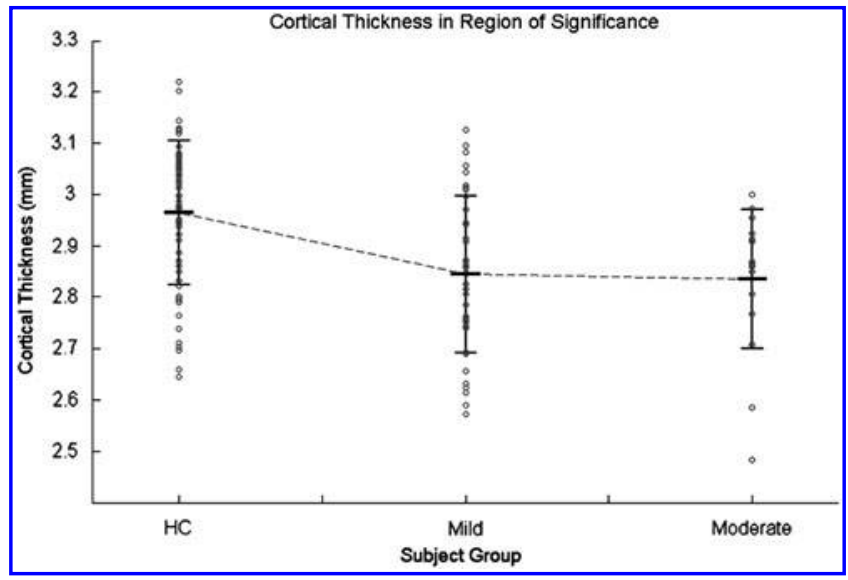

FIG. 3. Cortical thickness averaged over the region of interest defined by the statistically significant cluster between healthy controls (HC) and mild + moderate participants with traumatic brain injury (TBI). Each blue dot represents a subject and the average cortical thickness over this region. The mean and standard deviation error bars are displayed in black. ferior temporal, right insular, and right inferior frontal gyrus, associated with blast-related mild TBI. The moderate TBI groups showed similar localized findings in the right inferior temporal lobe, but to a smaller spatial extent. Our findings showed no differences in cortical thickness between mild and moderate TBI groups; thus, we were unable to detect a linear dose-response relationship as originally hypothesized.

Our results showed areas of significant cortical thinning that were lateralized and localized in nature. Based on the literature to date, it has been suggested that primary blast injury results in traumatic axonal injury and punctate microvascular disruptions. ${ }^{46-48}$ Because primary blast injury may also be accompanied by secondary and tertiary contact-based injury, however, it is possible that variations in injury mechanism are responsible for the lateralization and localization that was observed. There have been several articles in contact-based TBI that show injury in the orbitofrontal and anterior temporal lobe associated with the brain colliding with the sphenoid ridge and the free-edge of the tentorium cerebelli. ${ }^{9,45.46}$

As mentioned, despite a lack of positive findings on traditional neuroimaging studies, more than $50 \%$ of veterans meet the criteria for post-concussive disorder at long-term follow up. ${ }^{15}$ In the present study, TBI participants reported a greater number of postconcussive symptoms based on NSI total scores, which is consistent with the previous TBI literature. ${ }^{49,50}$ Further, the area of significant cortical thinning in the TBI population positively correlated with NSI scores, suggesting that post-concussive symptoms are related to right frontotemporal dysfunction. The NSI scale is not specific to TBI, however, because persons with affective disorders (i.e., PTSD) have been found to endorse higher symptom severity compared with persons with TBI alone.

Based on previous studies showing that cortical thinning is associated with several cognitive functions, we also examined relationships between cortical thickness values and neuropsychological measures. ${ }^{51,52}$ Our findings showed a low magnitude correlation between average cortical thickness values and a measure of verbal fluency skills (DKFEFS Verbal Fluency Condition 1); however, this finding was not statistically significant after Bonferroni correction. Other cognitive variables that were examined did not correlate significantly with cortical thickness in our sample.

These findings suggest that there are limited relationships between cortical thickness and cognitive functioning in this sample of veterans with chronic TBI. A possible explanation for this is that neuropsychological tests often assess cognitive functions requiring complex cortical and subcortical networks that may be disrupted differentially by a heterogeneous injury such as TBI. If present, cortical alterations that correspond to the neurocognitive measures used may have been too subtle to detect through the current SBM technique. 
The TBI groups demonstrated a high incidence of PTSD and depression symptoms, which is consistent with the previous literature. $^{2,53}$ The moderate TBI group had significantly higher scores on PCL-C and PHQ-9 compared with the mild TBI participants, suggesting a higher level of psychological stress. We also saw low magnitude correlations between cortical thickness and scores on measures of depression and PTSD symptoms; however, these correlations also were nonsignificant after Bonferroni correction.

Although blast exposure is considered to be the main contributor to initial injury, PTSD and depression may play a role in long-term cortical changes. Other studies have also suggested that PTSD affects cortical thickness independently of blast exposure. ${ }^{27,54}$ This is particularly pertinent in clinical evaluation and treatment of combat veterans, because PTSD has been shown to occur at a rate of more than four times as often in those with blast-related TBI compared with non-TBI veterans. ${ }^{2}$

It is clear that the impact of blast-related TBI versus comorbid psychiatric symptoms on cortical thickness represents an area for subsequent research and that the affected brain regions identified in this study may offer insights into this complex relationship. For example, the right inferior frontal, inferior temporal, and insular lobe have been shown to have strong connections to subcortical structures involved in PTSD, such as the amygdala and hippocampus, and thus may partially explain the relationship between TBI and PTSD in affecting cortical thickness. ${ }^{55}$

It is important to add that this study extends the investigation of Tate and coworkers, ${ }^{26}$ which analyzed mild blast-related TBI participants who were an average of 1 month post-injury. The results from Tate and associates ${ }^{26}$ showed left hemispheric regions of thinning in the superior temporal lobe and superior frontal lobe, which is clearly different from our findings of inferior temporal, right insular, and right inferior frontal gyrus thinning.

There are multiple reasons why variable results may have been obtained between our study and that of Tate and colleagues. ${ }^{26}$ These differences include: mean time since injury (1 month vs. 5 years), the number of subjects analyzed (22 vs. 113), and the designation of healthy controls (non-TBI veterans vs. healthy civilians). Our study also extended the work performed by Tate and coworkers, ${ }^{26}$ since they did not examine correlations between cortical thickness and measures of neuropsychological and psychological functioning.

\section{Limitations}

There are several limitations of this study that merit discussion. The SBM method only looks at cortical thickness and does not assess subcortical structures, some of which are expected to be associated with PTSD and TBI (e.g., amygdala and hippocampus). ${ }^{55}$ Another limitation is that blast-induced TBI is likely to be a heterogeneous injury and may not fully be elucidated by aggregated group difference studies. The participants recruited in this study included healthy controls and persons with blast-related TBI, but we did not assess military personnel having experienced combat without incurring a TBI. We cannot assess the effect of military training and combat on cortical thickness and how it affects the resulting regions of statistical significance. Further, we did not assess for duration or number of deployments in the military veteran group.

It also has recently been shown that early life interpersonal trauma positively correlated with both PTSD and cortical thinning in combat veterans. ${ }^{56}$ Early life trauma was not assessed in the present study, and therefore it is unclear how early life trauma contributes to our findings In addition, our assessment of post- concussive, PTSD, and depression symptoms was limited because we relied on retrospective self-report questionnaires, as opposed to evaluation and diagnosis by a clinician or using a structured interview format. Future research endeavors likely should use more thorough methods for evaluating PTSD and depression and their impact on cortical thickness in the context of brain injury.

\section{Conclusion}

This study provides evidence of cortical thinning after chronic blast-related mild or moderate TBI in military veterans and provides important insight into the natural progression of long-term cortical change after blast-related injury. We also found relationships between cortical thinning and aspects of cognitive and psychiatric functioning, including PTSD symptoms. Because of the high incidence of PTSD and depression symptoms in our sample, we cannot exclusively attribute cortical thinning to blast-related TBI. This sample, however, is highly representative of veterans with blastrelated injury, who often have comorbid psychiatric issues.

Our findings provided added insight into the potential factors affecting their symptom presentation, which has implications for diagnosis and treatment of this patient group. Future studies should be pursued to further examine the isolated effects of blast-induced TBI and PTSD on cortical thinning in comparison with healthy controls.

\section{Acknowledgments}

We would first like to thank the participants for taking part in this study. We would also like to thank D. Whitson, S. Taylor, and L. McDurmont for their work in recruitment and collection of data. This research was supported by the following grant from the Department of Defense: Evidence-Based Multimodal Neurodiagnostic Imaging of Traumatic Brain Injury and Post-Traumatic Stress Disorder. Contract Number W81XWH-08-2-0191, Richard Bucholz, Principal Investigator.

\section{Author Disclosure Statement}

No competing financial interests exist.

\section{References}

1. Terrio, H., Brenner, L.A., Ivins, B.J., Cho, J.M., Helmick, K., Schwab, K., Scally, K., Bretthauer, R., and Warden, D. (2009). Traumatic brain injury screening: preliminary findings in a US Army Brigade Combat Team. J. Head Trauma Rehabil. 24, 14-23.

2. Hoge, C.W., McGurk, D., Thomas, J.L., Cox, A.L., Engel, C.C., and Castro, C.A. (2008). Mild traumatic brain injury in U.S. Soldiers returning from Iraq. N. Engl. J. Med. 358, 453-463.

3. Morey, R.A., Haswell, C.C., Selgrade, E.S., Massoglia, D., Liu, C., Weiner, J., Marx, C.E.; MIRECC Work Group, Cernak, I., and McCarthy, G. (2013). Effects of chronic mild traumatic brain injury on white matter integrity in Iraq and Afghanistan war veterans. Hum. Brain Mapp. 34, 2986-2999.

4. Magnuson, J., Leonessa, F., and Ling, G.S. (2012). Neuropathology of explosive blast traumatic brain injury. Curr. Neurol. Neurosci. Rep. $12,570-579$

5. Saljo, A., Bolouri, H., Mayorga, M., Svensson, B., and Hamberger, A. (2010). Low-level blast raises intracranial pressure and impairs cognitive function in rats: prophylaxis with processed cereal feed. $\underline{\mathbf{J}}$. Neurotrauma 27, 383-389.

6. Taylor, P.A., and Ford, C.C. (2009). Simulation of blast-induced early-time intracranial wave physics leading to traumatic brain injury. J. Biomech. Eng. 131, 061007.

7. Elder, G.A., and Cristian, A. (2009). Blast-related mild traumatic brain injury: mechanisms of injury and impact on clinical care. Mt. Sinai J Med 76, 111-118. 
8. Taber, K.H., Warden, D.L., and Hurley, R.A. (2006). Blast-related traumatic brain injury: what is known? J. Neuropsych. Clin. Neurosci. $18,141-145$.

9. Bigler, E.D. (2007). Anterior and middle cranial fossa in traumatic brain injury: relevant neuroanatomy and neuropathology in the study of neuropsychological outcome. Neuropsychology 21, 515-531.

10. Barkhoudarian, G., Hovda, D.A., and Giza, C.C. (2011). The molecular pathophysiology of concussive brain injury. Clin. Sports Med. 30, 33-48, vii-iii.

11. Blyth, B.J., and Bazarian, J.J. (2010). Traumatic alterations in consciousness: traumatic brain injury. Emerg. Med. Clin. North Am. 28, 571-594.

12. Giza, C.C., and Hovda, D.A. (2001). The neurometabolic cascade of concussion. J. Athl. Train. 36, 228-235.

13. Jantzen, K.J. (2010). Functional magnetic resonance imaging of mild traumatic brain injury. J. Head Trauma Rehabil 25, 256-266.

14. McCrea, M., Iverson, G.L., McAllister, T.W., Hammeke, T.A., Powell, M.R., Barr, W.B., and Kelly, J.P. (2009). An integrated review of recovery after mild traumatic brain injury (MTBI): implications for clinical management. Clin. Neuropsychol. 23, 1368-1390.

15. Lange, R.T., Brickell, T.A., Ivins, B., Vanderploeg, R.D., and French, L.M. (2013). Variable, not always persistent, postconcussion symptoms after mild TBI in U.S. military service members: a five-year cross-sectional outcome study. J. Neurotrauma 30, 958-969.

16. Huang, M.X., Theilmann, R.J., Robb, A., Angeles, A., Nichols, S., Drake, A., D’Andrea, J., Levy, M., Holland, M., Song, T., Ge, S., Hwang, E., Yoo, K., Cui, L., Baker, D.G., Trauner, D., Coimbra, R., and Lee, R.R. (2009). Integrated imaging approach with MEG and DTI to detect mild traumatic brain injury in military and civilian patients. J. Neurotrauma 26, 1213-1226.

17. Davenport, N.D., Lim, K.O., Armstrong, M.T., and Sponheim, S.R. (2012). Diffuse and spatially variable white matter disruptions are associated with blast-related mild traumatic brain injury. NeuroImage 59, 2017-2024.

18. Dale, A.M., Fischl, B., and Sereno, M.I. (1999). Cortical surface-based analysis. I. Segmentation and surface reconstruction. NeuroImage 9 , 179-194.

19. Fischl, B., and Dale, A.M. (2000). Measuring the thickness of the human cerebral cortex from magnetic resonance images. Proc. Natl. Acad. Sci. U S A. 97, 11050-11055.

20. Fischl, B., Sereno, M.I., and Dale, A.M. (1999). Cortical surface-based analysis. II: Inflation, flattening, and a surface-based coordinate system. NeuroImage 9, 195-207.

21. Palacios, E.M., Sala-Llonch, R., Junque, C., Fernandez-Espejo, D., Roig, T., Tormos, J.M., Bargallo, N., and Vendrell, P. (2013). Longterm declarative memory deficits in diffuse TBI: correlations with cortical thickness, white matter integrity and hippocampal volume. Cortex 49, 646-657.

22. Turken, A.U., Herron, T.J., Kang, X., O'Connor, L.E., Sorenson, D.J., Baldo, J.V., and Woods, D.L. (2009). Multimodal surface-based morphometry reveals diffuse cortical atrophy in traumatic brain injury. BMC Med. Imaging 9, 20.

23. McCauley, S.R., Wilde, E.A., Merkley, T.L., Schnelle, K.P., Bigler, E.D., Hunter, J.V., Chu, Z., Vasquez, A.C., and Levin, H.S. (2010). Patterns of cortical thinning in relation to event-based prospective memory performance three months after moderate to severe traumatic brain injury in children. Dev. Neuropsychol. 35, 318-332.

24. Merkley, T.L., Bigler, E.D., Wilde, E.A., McCauley, S.R., Hunter, J.V., and Levin, H.S. (2008). Diffuse changes in cortical thickness in pediatric moderate-to-severe traumatic brain injury. J. Neurotrauma $25,1343-1345$

25. Wilde, E.A., Merkley, T.L., Bigler, E.D., Max, J.E., Schmidt, A.T., Ayoub, K.W., McCauley, S.R., Hunter, J.V., Hanten, G., Li, X., Chu, Z.D., and Levin, H.S. (2012). Longitudinal changes in cortical thickness in children after traumatic brain injury and their relation to behavioral regulation and emotional control. Int. J. Dev. Neurosci. 30 267-276.

26. Tate, D.F., York, G.E., Reid, M.W., Cooper, D.B., Jones, L., Robin, D.A., Kennedy, J.E., and Lewis, J. (2014). Preliminary findings of cortical thickness abnormalities in blast injured service members and their relationship to clinical findings. Brain Imaging Behav. 8, 102-109.

27. Lindemer, E.R., Salat, D.H., Leritz, E.C., McGlinchey, R.E., and Milberg, W.P. (2013). Reduced cortical thickness with increased lifetime burden of PTSD in OEF/OIF Veterans and the impact of comorbid TBI. NeuroImage Clin. 2, 601-611.
28. Cooper, D.B., Chau, P.M., Armistead-Jehle, P., Vanderploeg, R.D., and Bowles, A.O. (2012). Relationship between mechanism of injury and neurocognitive functioning in OEF/OIF service members with mild traumatic brain injuries. Mil. Med. 177, 1157-1160.

29. Hudak, A., Warner, M., Marquez de la Plata, C., Moore, C., Harper, C., and Diaz-Arrastia, R. (2011). Brain morphometry changes and depressive symptoms after traumatic brain injury. Psychiatry Res. 191, $160-165$.

30. Konrad, C., Geburek, A.J., Rist, F., Blumenroth, H., Fischer, B., Husstedt, I., Arolt, V., Schiffbauer, H., and Lohmann, H. (2011). Long-term cognitive and emotional consequences of mild traumatic brain injury. Psychol. Med. 41, 1197-1211.

31. Huang, M.X., Nichols, S., Robb, A., Angeles, A., Drake, A., Holland, M., Asmussen, S., D'Andrea, J., Chun, W., Levy, M., Cui, L., Song, T., Baker, D.G., Hammer, P., McLay, R., Theilmann, R.J., Coimbra, R., Diwakar, M., Boyd, C., Neff, J., Liu, T.T., WebbMurphy, J., Farinpour, R., Cheung, C., Harrington, D.L., Heister, D., and Lee, R.R. (2012). An automatic MEG low-frequency source imaging approach for detecting injuries in mild and moderate TBI patients with blast and non-blast causes. NeuroImage 61, 10671082 .

32. Luo, Q., Xu, D., Roskos, T., Stout, J., Kull, L., Cheng, X., Whitson, D., Boomgarden, E., Gfeller, J., and Bucholz, R.D. (2013). Complexity analysis of resting state magnetoencephalography activity in traumatic brain injury patients. J. Neurotrauma 30, 1702-1709.

33. Dickerson, B.C., Fenstermacher, E., Salat, D.H., Wolk, D.A., Maguire, R.P., Desikan, R., Pacheco, J., Quinn, B.T., Van der Kouwe, A., Greve, D.N., Blacker, D., Albert, M.S., Killiany, R.J., and Fischl, B. (2008). Detection of cortical thickness correlates of cognitive performance: reliability across MRI scan sessions, scanners, and field strengths. NeuroImage 39, 10-18.

34. Management of Concussion/m, T.B.I.G. (2009). VA/DoD Clinical Practice Guideline for Management of Concussion/Mild Traumatic Brain Injury. J. Rehabil. Res. Devel. 46, CP1-68.

35. Weschsler, D. (1997). WAIS-III Administration and Scoring Manual. San Antonio, TX.

36. (1944). Army Individual Test Battery, in: Manual of Directions and Scoring. War Department, Adjutant General's Office: Washington, DC.

37. Delis, D.C., Kramer, J. H., Kaplan, E., and Ober, B. A. (2000). California Verbal Learning Test Manual: Second Edition, Adult Version. Psychological Corporation: San Antonio, Texas.

38. Delis, D.C., Kramer, J. H., Kaplan, E., \& Ober, B. A. (2001). DelisKaplan Executive Function System: D-KEFS. Psychological Corporation: Orlando, FL.

39. Cicerone, K., and Kalmar, K. (1995). Persistent postconcussion syndrome: The structure of subjective complaints after mild traumatic brain injury. J Head Trauma Rehabil. 10, 1-17.

40. Kroenke, K., Spitzer, R.L., and Williams, J.B. (2001). The PHQ-9: validity of a brief depression severity measure. J. Gen. Intern. Med. $16,606-613$.

41. Weathers, F.W., Litz, B. T., Herman, D. S., Huska, J. A., and Keane, T. M. (1993). The PTSD Checklist (PCL): reliability, validity, and diagnostic utility, in: Annual Convention of the International Society for Traumatic Stress Studies. San Antonio: International Society for Traumatic Stress Studies (vol. 141).

42. Fischl, B., Salat, D.H., Busa, E., Albert, M., Dieterich, M., Haselgrove, C., van der Kouwe, A., Killiany, R., Kennedy, D., Klaveness, S., Montillo, A., Makris, N., Rosen, B., and Dale, A.M. (2002). Whole brain segmentation: automated labeling of neuroanatomical structures in the human brain. Neuron 33, 341-355.

43. Segonne, F., Dale, A.M., Busa, E., Glessner, M., Salat, D., Hahn, H.K., and Fischl, B. (2004). A hybrid approach to the skull stripping problem in MRI. NeuroImage 22, 1060-1075.

44. Desikan, R.S., Segonne, F., Fischl, B., Quinn, B.T., Dickerson, B.C., Blacker, D., Buckner, R.L., Dale, A.M., Maguire, R.P., Hyman, B.T., Albert, M.S., and Killiany, R.J. (2006). An automated labeling system for subdividing the human cerebral cortex on MRI scans into gyral based regions of interest. NeuroImage 31, 968-980.

45. Fischl, B., Sereno, M.I., Tootell, R.B., and Dale, A.M. (1999). Highresolution intersubject averaging and a coordinate system for the cortical surface. Hum. Brain Mapp. 8, 272-284.

46. Fischl, B., Salat, D.H., van der Kouwe, A.J., Makris, N., Segonne, F., Quinn, B.T., and Dale, A.M. (2004). Sequence-independent segmentation of magnetic resonance images. NeuroImage 23 Suppl 1, S69-S84. 
47. Hagler, D.J., Jr., Saygin, A.P., and Sereno, M.I. (2006). Smoothing and cluster thresholding for cortical surface-based group analysis of fMRI data. NeuroImage 33, 1093-1103.

48. Bhattacharjee, Y. (2008). Neuroscience. Shell shock revisited: solving the puzzle of blast trauma. Science $319,406-408$.

49. King, P.R., Donnelly, K.T., Donnelly, J.P., Dunnam, M., Warner, G., Kittleson, C.J., Bradshaw, C.B., Alt, M., and Meier, S.T. (2012). Psychometric study of the Neurobehavioral Symptom Inventory. $\underline{\mathrm{J}}$. Rehabil. Res. Devel. 49, 879-888.

50. Soble, J.R., Silva, M.A., Vanderploeg, R.D., Curtiss, G., Belanger, H.G., Donnell, A.J., and Scott, S.G. (2014). Normative Data for the Neurobehavioral Symptom Inventory (NSI) and post-concussion symptom profiles among TBI, PTSD, and nonclinical samples. Clin. Neuropsychol. 28, 614-632.

51. Christopoulos, G.I., Tobler, P.N., Bossaerts, P., Dolan, R.J., and Schultz, W. (2009). Neural correlates of value, risk, and risk aversion contributing to decision making under risk. J. Neurosci. 29, 12574-12583.

52. White, S.F., Costanzo, M.E., Blair, J.R., and Roy, M.J. (2015). PTSD symptom severity is associated with increased recruitment of topdown attentional control in a trauma-exposed sample. NeuroImage Clin. 7, 19-27.

53. Brenner, L.A., Vanderploeg, R.D., and Terrio, H. (2009). Assessment and diagnosis of mild traumatic brain injury, posttraumatic stress disorder, and other polytrauma conditions: burden of adversity hypothesis. Rehabil. Psychol. 54, 239-246.

54. Woodward, S.H., Schaer, M., Kaloupek, D.G., Cediel, L., and Eliez, S. (2009). Smaller global and regional cortical volume in combatrelated posttraumatic stress disorder. Arch. Gen. Psychiatry 66, 1373-1382.

55. Liberzon, I., and Martis, B. (2006). Neuroimaging studies of emotional responses in PTSD. Ann. N Y Acad. Sci. 1071, 87-109.

56. Corbo, V., Salat, D.H., Amick, M.M., Leritz, E.C., Milberg, W.P., and McGlinchey, R.E. (2014). Reduced cortical thickness in veterans exposed to early life trauma. Psychiatry Res. 223, 53-60.

Address correspondence to: Alex P. Michael, MD

Division of Neurosurgery

Southern Illinois University School of Medicine 421 N. 9th Street Springfield, IL 62702

E-mail: amichael@siumed.edu 


\section{This article has been cited by:}

1. Savjani Ricky R., Taylor Brian A., Acion Laura, Wilde Elisabeth A., Jorge Ricardo E.. 2017. Accelerated Changes in Cortical Thickness Measurements with Age in Military Service Members with Traumatic Brain Injury. Journal of Neurotrauma 34:22, 3107-3116. [Abstract] [Full Text HTML] [Full Text PDF] [Full Text PDF with Links]

2. Catherine D. Chong, Visar Berisha, Chia-Chun Chiang, Katherine Ross, Todd J. Schwedt. 2017. Less Cortical Thickness in Patients With Persistent Post-Traumatic Headache Compared With Healthy Controls: An MRI Study. Headache: The Journal of Head and Face Pain 16. . [Crossref]

3. Kaplan Gary B., Leite-Morris Kimberly A., Wang Lei, Rumbika Kendra K., Heinrichs Stephen C., Zeng Xiang, Wu Liquan, Arena Danielle T., Teng Yang D.. Pathophysiological Bases of Comorbidity: Traumatic Brain Injury and Post-Traumatic Stress Disorder. Journal of Neurotrauma, ahead of print. [Abstract] [Full Text HTML] [Full Text PDF] [Full Text PDF with Links]

4. Ming-Liang Wang, Xiao-Er Wei, Meng-Meng Yu, Peng-Yang Li, Wen-Bin Li. 2017. Self-reported traumatic brain injury and in vivo measure of $\mathrm{AD}$-vulnerable cortical thickness and $\mathrm{AD}$-related biomarkers in the ADNI cohort. Neuroscience Letters 655, 115-120. [Crossref]

5. David H. Salat, Meghan E. Robinson, Danielle R. Miller, Dustin C. Clark, Regina E. McGlinchey. 2017. Neuroimaging of deployment-associated traumatic brain injury (TBI) with a focus on mild TBI (mTBI) since 2009. Brain Injury 31:9, 1204-1219. [Crossref]

6. Spitz Gershon, Alway Yvette, Gould Kate Rachel, Ponsford Jennie L.. 2017. Disrupted White Matter Microstructure and Mood Disorders after Traumatic Brain Injury. Journal of Neurotrauma 34:4, 807-815. [Abstract] [Full Text HTML] [Full Text PDF] [Full Text PDF with Links] [Supplemental Material]

7. Eduardo Gonz?lez-Toledo, Nicol?s Fayed Miguel, Laura Viguera, Kanika Sharma, Piyush Kalakoti, Navdeep Samra, Anil Nanda, Hai Sun. Utility of Magnetic Resonance Findings in Elucidating Structural and Functional Brain Impairment in Traumatic Brain Injury 443-456. [Crossref]

8. Ming-Liang Wang, Wen-Bin Li. 2016. Cognitive impairment after traumatic brain injury: The role of MRI and possible pathological basis. Journal of the Neurological Sciences 370, 244-250. [Crossref]

9. Erin D. Bigler, Brandon A. Zielinski, Naomi Goodrich-Hunsaker, Garrett M. Black, B. S. Trevor Huff, Zachary Christiansen, Dawn-Marie Wood, Tracy J. Abildskov, Maureen Dennis, H. Gerry Taylor, Kenneth Rubin, Kathryn Vannatta, Cynthia A. Gerhardt, Terry Stancin, Keith Owen Yeates. 2016. The Relation of Focal Lesions to Cortical Thickness in Pediatric Traumatic Brain Injury. Journal of Child Neurology 31:11, 1302-1311. [Crossref]

10. Maya Troyanskaya, Nicholas J. Pastorek, Randall S. Scheibel, Nancy J. Petersen, Annette Walder, Helene K. Henson, Harvey S. Levin. 2016. Choosing appropriate comparison group participants in studies of veterans: Characteristics of orthopedically injured and uninjured Operation Enduring Freedom/Operation Iraqi Freedom/Operation New Dawn veterans. Journal of Clinical and Experimental Neuropsychology 38:7, 811-819. [Crossref]

11. Aric F. Logsdon, Brandon P. Lucke-Wold, Linda Nguyen, Rae R. Matsumoto, Ryan C. Turner, Charles L. Rosen, Jason D. Huber. 2016. Salubrinal reduces oxidative stress, neuroinflammation and impulsive-like behavior in a rodent model of traumatic brain injury. Brain Research 1643, 140-151. [Crossref]

12. Wei Lin, Li-kun Yang, Sang Cai, Jie Zhu, Yi Feng, Li-xiang Yang, Zhi-zhong Feng, Pei-pei Li, Jun-hui Chen, Yu-hai Wang. 2016. Cognitive function and biomarkers after traumatic brain injury: protocol for a prospective inception cohort study. Asia Pacific Journal of Clinical Trials: Nervous System Diseases 1:4, 170. [Crossref] 